American J. of Engineering and Applied Sciences 4 (1): 169-174, 2011

ISSN 1941-7020

(C) 2010 Science Publications

\title{
Design and Manufacturing of a Custom Skull Implant
}

\author{
${ }^{1}$ Juan Felipe Isaza Saldarriaga, ${ }^{1}$ Santiago Correa Vélez, \\ ${ }^{2}$ M.D. Adolfo Cumplido Posada, ${ }^{3}$ I.E. Balmore Bedoya Henao \\ and ${ }^{3}$ M.E. Carlos Andrés Torres Valencia \\ ${ }^{1}$ Department of Product Design Engineering, Grupo, \\ De Investigación en Bioingeniería GIB, EAFIT-CES Universities, Medellin, Colombia \\ ${ }^{2}$ Faculty of Medicine, University of Antioquia, Medellín, Colombia \\ ${ }^{3}$ Department of Desing and Prototyping, Indústrias Médicas Sampedro S.A., Medellín, Colombia
}

\begin{abstract}
Problem statement: Cranioplasty is defined as a neurosurgical procedure to cover an injured bone in the skull. This procedure is carried out in order to protect and restore intracranial structures and to restore the appearance and psychological stability of the patient. Advances in medical imaging, such as MRI and CT, have allowed the 3D reconstruction of anatomical structures for several medical applications, including the design of custom-made implants. This study describes the methodology used to design a custom-made cranial implant for a 13-year-old patient who suffered a lesion in the left frontoparietal region of the skull caused by a fall. Approach: The design of the implant was based on the 3D reconstruction of the skull of the patient, obtained by a CT scan, using Rapid Form ${ }^{\circledR}$ 2006. Once the preliminary design was completed, 3D models of the injured region of the skull and of the implant were fabricated in a Rapid Prototyping (RP) machine using Fused Deposition Modeling Technology (FDM) with the purpose of functionally and dimensionally validating the implant. Subsequently, the implant was fabricated using a 1.2-mm-thick Titanium Alloy (Ti6Al4V) plate. Results: The prosthesis was successfully implanted. The surgical time was $85 \%$ shorter than that for the same type of surgery in which standard commercial implants and titanium meshes are used. This decrease in surgery time is primarily the result of eliminating the need for trial and error procedures to achieve a good fit for the implant. Finally, the appearance of the patient was restored, allowing the patient to safely perform daily activities. Conclusion: The use of 3D reconstruction techniques from medical images reduces the possibility of errors during surgery, improves fit and provides better implant stability. The use of 3D models designed in RP proved to be an effective practice in the design process.
\end{abstract}

Key words: 3D reconstruction, Electron Beam Melting (EBM), Computer Numerical Control Machines (CNC), reverse engineering biomaterials, Rapid Prototyping (RP), Fused Deposition Modeling Technology (FDM), Polymethylmethacrylate (PMMA)

\section{INTRODUCTION}

Cranioplasty is defined as a neurosurgical procedure to cover an injured bone in the skull. Such injuries can be caused by congenital defects, diseases, accidents, infections or tumors. This procedure is carried out in order to protect and restore intracranial structures and to restore the appearance and psychological stability of the patient (Joffe et al., 1999). The success of reconstructive skull surgery depends on the preoperative evaluation of the defect, the design and manufacturing of the implant and the execution of the surgical procedure. Advances in medical imaging, such as MRI and CT, have allowed the 3D reconstruction of anatomical structures for several medical applications, including the design of custom-made implants. In the case of cranial implants, several studies have reported the advantages of using different computer-aided design and advance manufacturing platforms (CAD/CAM) (Drstvensek et al., 2008). These advantages are reflected in the better fit of the implant, a decrease in the surgery time and better esthetic results (Joffe et al., 1992; Eufinger et al., 1995; Heissler et al., 1998; Mazzoli et al., 2009) compared with manual methods

Corresponding Author: Juan Felipe Isaza, Department of Product Design Engineering, EAFIT University, Medellín, Colombia. Grupo de Investigación en Bioingeniería GIB, EAFIT-CES Tel: (574) 261-9500 
that require longer design and manufacturing times, the success of which largely depends on the skill of the medical sculptor.

Throughout history, different materials have been used in the manufacture of cranial implants (Neovius and Engstrand, 2009; Artico et al., 2003) among the most common are (i) acrylics such as Polymethylmethacrylate (PMMA) (Malis, 1989; Yanai, 1991; Gronet et al., 2003; )( Joffe et al., 1992) (ii) implants designed from bone grafts (Santoni-Rugiu, 1969; Kulali and Kayaale, 1991; Psillakis et al., 1979; Prolo and Oklund, 1984; Osawa et al., 1990) (iii) ceramic materials such as hydroxyapatite (Hollier and Stal, 2004). Furthermore, in the group of biocompatible metals, titanium alloys are highlighted (Koppel et al., 1999; Kuttenberger and Hardt, 2001). In this study, a plate of biocompatible titanium alloy (Ti6Al4V) was used for the construction of the implant. With the methodology used, the prosthesis was successfully implanted. The surgical time decreased by $85 \%$ and the appearance of the patient was restored, allowing the patient to safely perform daily activities.

\section{MATERIALS AND METHODS}

Clinical description of the patient: The patient was a 13-year-old male who suffered a fall from a height of approximately $1.6 \mathrm{~m}$. As a result, he experienced a cranioencephalic trauma that generated a left acute epidural hematoma. The mortality rate for this type of injury is about $40 \%$. As the hematoma grows, it moves the brain away from the brain stem, causing severe headache, a decrease in the contralateral force, confusion or coma, dilated pupils (mydriasis), respiratory rhythm disturbances and ultimately, death. For this reason, it was necessary to perform a craniotomy in order to remove or drain the hematoma and aid the clogging of the arteries that were broken to prevent bleeding.

The patient was referred to neurosurgery. The bone flap was removed to allow expansion of the brain in case cerebral edema had developed. The portion of skull removed was approximately $68.7 \mathrm{~cm}^{2}$. Thus, the brain was only protected by the meanings and the scalp in this region; this situation represented a high risk of severe damage even with minimal impact (Fig. 1). The recovery of the patient after this procedure was excellent.

After a reasonable time, when the risk of edema was over, a cranioplasty was performed. To support this procedure, a CT scan of the patient was completed and a titanium alloy (Ti6Al4V) was used to manufacture the implant; this material was chosen because of its high degree of biocompatibility and mechanical resistance.

3D Reconstruction: For the 3D reconstruction of the injured area, a CT scan of the head and neck was used.
The scan consisted of 751 images with a distance between cuts of $0.3 \mathrm{~mm}$. This procedure was conducted in RapidForm ®2006 (Inus Technology, Seoul, South Korea). As a first step, a 3D reconstruction of the skull was performed, with the purpose of observing the injury (Fig. 2).

Subsequently, a 3D reconstruction of the skin was performed to clearly observe the asymmetry caused by the injury, which compromised the appearance of the patient (Fig. 3).

Geometric modeling of the implant: Once a 3D reconstruction of the relevant anatomy was obtained, a symmetry plane that coincided with the sagittal plane of the patient was created. From the sagittal plane, the skull geometry was divided in two halves. The injury was located in the left half (Fig. 4).

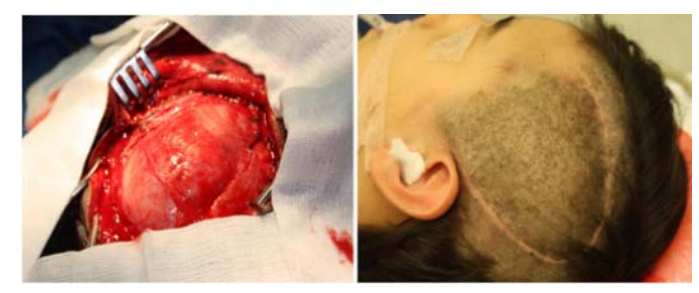

Fig.1: Brain protected by the meninges and the scalp

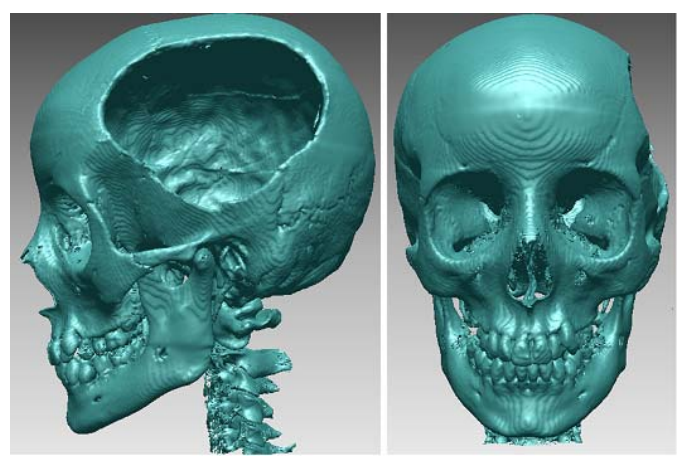

Fig. 2: 3D reconstruction of the skull

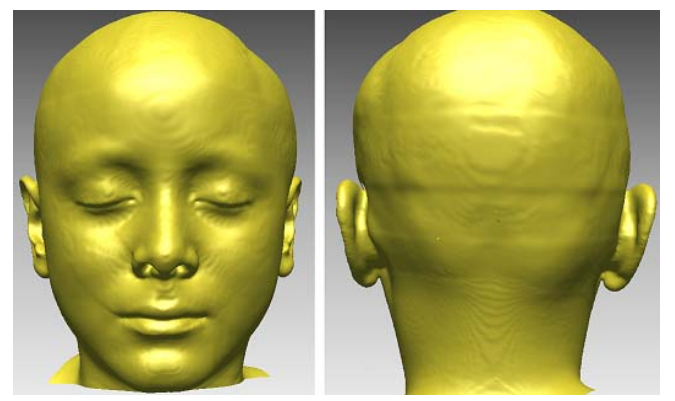

Fig. 3: Asymmetry caused by the injury 


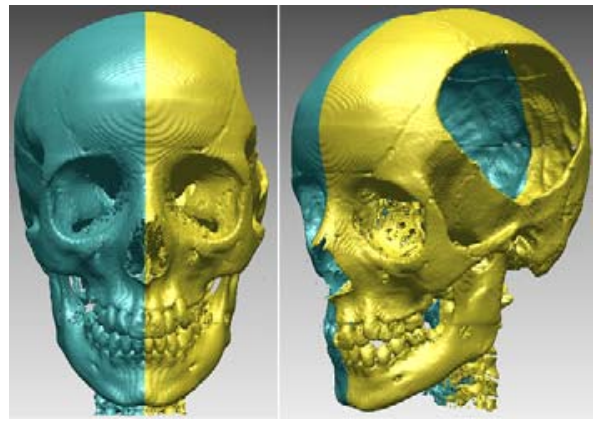

Fig. 4: Skull geometry divided in two halves

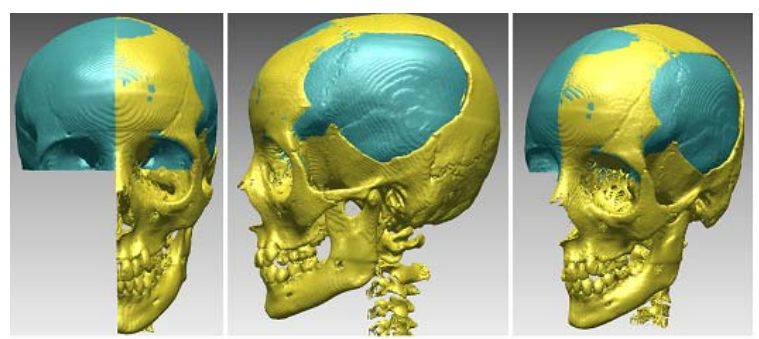

Fig. 5:Mirror operation performed on the healthy half of the skull

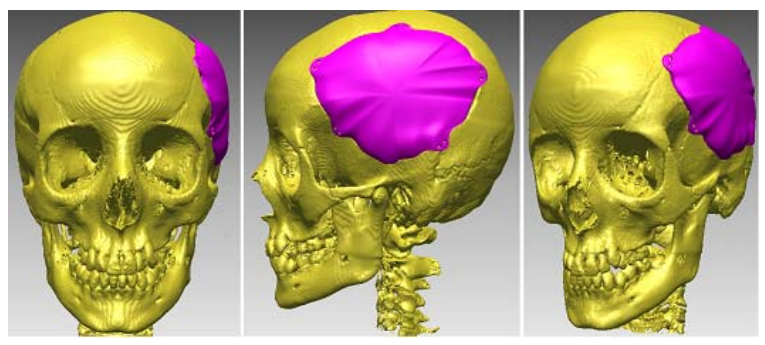

Fig. 6: Geometric modeling of the implant

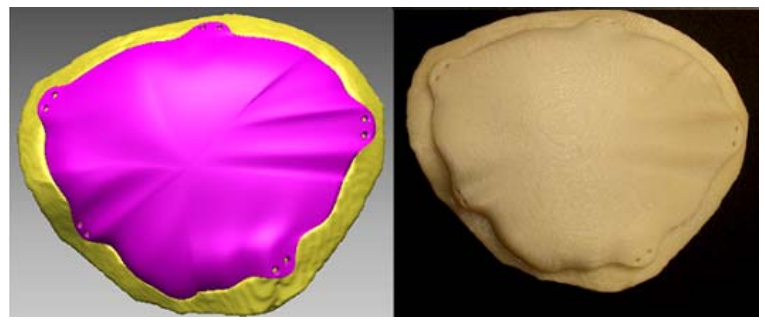

Fig. 7:Dimensional and functional validation of the implant

Thereafter, using the sagittal plane as the symmetry plane, a mirror operation was performed on the healthy half of the skull, which was used as the basis of the geometric modeling of the implant (Fig. 5). On the skull surface, adjacent to the periphery of the injury, five areas of support were defined and these areas were used as assembly regions between the implant and the skull using 10 mini screws (Fig. 6).

Dimensional and functional validation of the implant: In a Rapid Prototyping (RP) machine, using Fused Deposition Modeling technology (FDM), models of the implant and a portion of the injury were manufactured. To reduce the cost and manufacturing time, only a small portion of the skull injury was fabricated (Fig. 7).

Manufacturing of the implant: Using a $1.2 \mathrm{~mm}$ thick titanium alloy (Ti6Al4V) plate, the implant was fabricated. The manufacturing process was carried out by applying pressure on the titanium plate, which was exerted by two pieces of Duraluminium in which the outer 3D surface of the implant had been machined. Finally, the holes for inserting the mini screws were machined.

\section{RESULTS}

A custom-made, titanium alloy (Ti6Al4V) implant was obtained. Fig. 8 shows the final appearance of the implant and its dimensional and functional verification using the portion of the skull previously fabricated in RP. The weight of the implant was $66.33 \mathrm{~g}$. The approximate volume of the portion of skull that was removed was $19.7 \mathrm{~cm}^{3}$. Assuming that the density of bone is $\rho=210 \mathrm{~kg} \mathrm{~m}^{-3}$ (Margulies and Thibault, 2000), an approximate weight of $42.35 \mathrm{~g}$ was obtained; therefore, the weight of the implant was 1.6 times greater than the portion of bone removed.

Implantation process: As a first step, the implant was sterilized with ethylene oxide. After sterilization, the implant was successfully placed on the skull. Modifications to the implant, such as folds or removal of material, were not needed (Fig. 9). Once the implant was in place, the holes to insert the mini screws were made. Finally, the patient was sutured. The entire implantation process lasted about 45 min the surgery time was reduced by $85 \%$ compared with surgeries in which commercial implants and titanium meshes were used.

Patient monitoring: After four months, a control follow-up was performed. The patient was very satisfied with the cosmetic result and the security offered by the implant. The surgical scar was healthy and no pain, inflammation or rejection of the material was observed. A new CT scan demonstrated that the implant was in an excellent position and no further complications were expected (Fig. 10). 


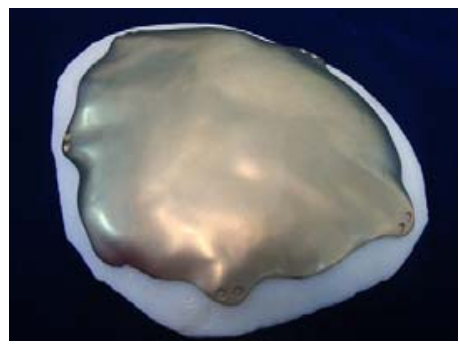

Fig. 8: Custom-made implant

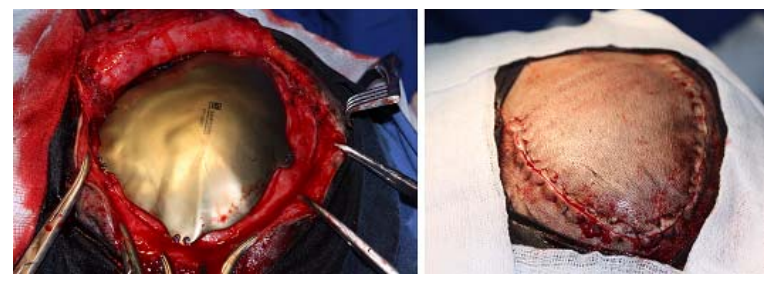

Fig. 9: Cranioplasty

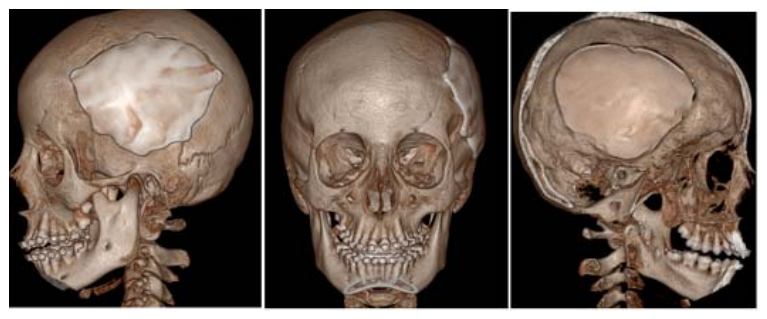

Fig. 10: Patient postoperative after four months

\section{CONCLUSION}

A custom-made cranial implant was designed, fabricated and placed into a patient whose brain was only protected by the meninges and the scalp after a $1.6 \mathrm{~m}$ fall. This situation represented a high risk for his health. After surgery, the appearance of the patient was restored, allowing the patient to safely perform daily activities.

Typical cranioplasty procedures, in which standard implants and titanium meshes are used, last approximately 4-5 $\mathrm{h}$ due to the trial and error procedures that are implemented during the surgery in order to achieve a good fit of the implant. With our methodology, a decrease in the surgery time of approximately $85 \%$ was obtained. This reduction in time also reduces the risk for the patient and reduces surgery costs. The use of 3D reconstruction techniques from medical images reduces the possibility of errors during surgery, improves fit and provides better implant stability after fixation with mini screws (Singare et al., 2004; Eufinger et al., 1995). The dimensional and functional verification of the implant using 3D models designed in RP proved to be an effective practice in the design process (D'urso et al., 1999;1998; 2000) because during the surgery modifications of the implant or the skull region around the injury were not necessary. The models were also an effective communication tool for the neurosurgeon, the patient and the family of the patient when discussing the surgical procedure (Petzold et al., 1999; Webb, 2000; Sailer et al., 1998).

The Titanium Alloy (Ti6Al4V) used to manufacture the implant proved to be a suitable material for this type of application due to its biocompatibility and good mechanical properties (Winder et al., 1999; Joffe et al., 1999; Heissler et al., 1998; Mazzoli et al., 2009; Mottaran et al., 2004). This is demonstrated by the absence of postoperative infections and the proper healing and restoration of normal activities of the patient without any medical complications. The process used to manufacture the implant was less expensive and faster than other high-cost technologies, such as Electron Beam Melting (EBM) (Mazzoli et al., 2009) and traditional machining methods using Computer Numerical Control Machines (CNC), in which large amounts of titanium are wasted. If a casting process had been used, it would have been necessary to make a mold and have access to a furnace that reached temperatures up to $1600^{\circ} \mathrm{C}$ (Heissler et al., 1998).

An implant was constructed with a weight increase of $57 \%$ relative to the weight of the portion of the skull removed. In future research, the weight of the implant could be made closer to the weight of the portion of skull using thinner plates. According to Joffe et al., implants made from $0.72 \mathrm{~mm}$ thick plates were successful. Once the CT scan had been completed, the design and manufacturing process was carried out in 10 days. This situation makes this procedure highly competitive with other commercial alternatives due to the time and cost required to import an implant into Colombia and Latin America.

\section{REFERENCES}

Artico, M., L. Ferrante, F.S. Pastore, E.O. Ramundo and D. Cantarelli et al., 2003. Bone autografting of the calvaria and craniofacial skeleton: Historical background, surgical results in a series of 15 patients, and review of the literature. Surgical Neurol., 60: 71-79. PMID: 12865021

Drstvensek, I., N.I. Hren, T. Strojnik, T. Brajlih and B. Valentan et al., 2008. Applications of rapid prototyping in cranio-maxilofacial surgery procedures. Int. J. Biol. Biomed. Eng., 2: 29-38. 
Am. J. Engg. \& Applied Sci., 4 (1): 169-174, 2011

D'urso, P.S., D.J. Effeney, W.J. Earwaker, T.M. Barker and M.J. Redmond et al., 2000. Custom cranioplasty using stereolithography and acrylic. British J. Plastic Surg., 53: 200-204. DOI: 10.1054/bjps.1999.3268

D'urso, P.S., R.L. Atkinson, J. Bruce, D.J. Effeney and M.W. Lanigan et al., 1998. Stereolithographic (SL) biomodelling in craniofacial surgery. British J. Plastic Surg., 51: 522-530.

D'urso, P.S., T.M. Barker, W.J. Earwaker, L.J. Bruce and R.L. Atkinson et al., 1999. Stereolithographic biomodelling in cranio-maxillofacial surgery: a prospective trial. J. Cranio Maxillofacial Surg., 27: 30-37. DOI: 10.1016/S1010-5182(99)80007-9

Eufinger, H., M. Wehmoller, E. Machtens, L. Heuser and A. Harders et al., 1995. Reconstruction of craniofacial bone defects with individual alloplastic implants based on CAD/CAM-manipulated CTdata. J. Craniomaxillofac Surg., 23: 175- 181. DOI: 10.1016/S1010-5182(05)80007-1

Gronet, P.M., G.A. Waskewicz and C. Richardson, 2003. Preformed acrylic cranial implants using fused deposition modeling: a clinical report. J. Prosthet Dent, 90: 429-433. DOI: 10.1016/j.prosdent.2003.08.023

Heissler, E., F.S. Fischer, S. Bolouri, T. Lehrnann and W. Mathar et al., 1998. Custom-made cast titanium implants produced with CAD/CAM for the reconstruction of cranium defects. Int. J. Oral Maxillofac Surg., 27: 334-338. DOI: 10.1016/S0901-5027(98)80060-X

Hollier, L.H. and S. Stal, 2004. The use of hydroxyapatite cements in craniofacial surgery. Clin Plastic Surg., 31: 423-428. PMID: 15219749

Joffe, J.M., P.J.C. Mcdermott, A.D. Linney, C.A. Mosse and M. Harris, 1992. Computer-generated titanium cranioplasty: Report of a new technique for repairing skull defects. Br. J. Neurosurg, 6: 343-350.

Joffe, J.M., S.R. Nicoll, R. Richards, A.D. Linney and M. Harris, 1999. Validation of computer-assisted manufacture of titanium plates for cranioplasty. Int. J. Oral Maxillofac. Surg., 28: 309-313. DOI: 10.1016/S0901-5027(99)80165-9

Koppel, D.A., K.F. Moos and F.S. Walker, 1999. Skull reconstruction with a two-part interlocking custommade titanium plate. Br J. Oral. Maxillofac Surg., 37: 70-72. DOI: 10.1054/bjom.1998.0349

Kulali, A. and S. Kayaale, 1991. Single-table autogenous calvarial grafting for cranioplasty. J. Craniomaxillofac Surg., 19: 208- 211. DOI: 10.1016/S1010-5182(05)80549-9

Kuttenberger, J.J. and N. Hardt, 2001. Long-term results following reconstruction of craniofacial defects with titanium micro-mesh systems. J. Craniomaxillofac Surg., 29: 75-81. DOI: 10.1054/jcms.2001.0197
Malis, L., 1989. Titanium mesh and acrylic cranioplasty. Neurosurg, 25: 351-355. PMID: 2671787

Margulies, S.S. and K.L. Thibault, 2000. Infant skull and suture properties: Measurements and implications for mechanisms of pediatric brain injury. J. Biomech. Eng., 122: 364-371. DOI: 10.1115/1.1287160

Mazzoli, A., M. Germani and R. Raffaeli, 2009. Direct fabrication through electron beam melting technology of custom cranial implants designed in a PHANToM-based haptic environment. Mater. Design, $\quad 30$ : 3186-3192. DOI: 10.1016/j.matdes.2008.11.013

Mottaran, R., L. Guarda-Nardini, S. Fusetti, G. Ferronet and G. Salar, 2004. Reconstruction of a large posttraumatic cranial defect with a customized titanium plaque. J. Neurosurg Sci., 48: 143-147. PMID: 15557886

Neovius, E. and T. Engstrand, 2009. Craniofacial reconstruction with bone and biomaterials: review over the last 11 years. J. Plastic, Reconstr. Aesthet. Surg., 63: 1615-1623. PMID: 19577527

Osawa, M., H. Hara, Y. Ichinose, T. Koyama and S. Kobayashi et al., 1990. Cranioplasty with a frozen and autoclaved bone flap. Acta Neurochir, 102: 38-41. DOI: $10.1007 / \mathrm{BF} 01402184$

Petzold, R., H.F. Zeilhofer and W.A. Kalender, 1999. Rapid prototyping technology in medicine-basics and applications. Computerized Med. Imag. Graphics, 23: 277-284.

Prolo, D.J. and S. Oklund, 1984. Composite autogeneic human cranioplasty: Frozen skull supplemented with fresh iliac corticocancellous bone. Neurosurgery, 15: 846-851. PMID: 6514157

Psillakis, J.M., V.L. Nocchi and S.A. Zanini, 1979. Repair of large defect of frontal bone with free graft of outer table of parietal bones. Plast Reconstr Surg., 64: 827-30. PMID: 390579

Sailer, H.F., P.E. Haers, C.P.E. Zollikofer, T. Warnke and F.R. Carls et al., 1998. The value of stereolithographic models for preoperative diagnosis of craniofacial deformities and planning of surgical corrections. Int. J. Oral Maxillofac, 27: 327-333. DOI: 10.1016/S0901-5027(98)80059-3

Santoni-Rugiu, P., 1969. Repair of skull defects by outer table osteoperiosteal free grafts. Plast Reconstr Surg., 43: 157-61.

Singare, S., L. Dichen, L. Bingheng, L. Yanpu and G. Zhenyu et al., 2004. Design and fabrication of custom mandible titanium tray based on rapid prototyping. Med. Eng. Phys., 26: 671-676. DOI: 10.1016/j.medengphy.2004.06.001

Webb, P.A., 2000. A review of Rapid Prototyping (RP) techniques in the medical and biomedical sector. J. Med. Eng. Technol., 24: 149-153. PMID: 11105287 
Am. J. Engg. \& Applied Sci., 4 (1): 169-174, 2011

Winder, J., R.S. Cooke, J. Gray, T. Fannin and T. Fegan, 1999. Medical rapid prototyping and 3D CT in the manufacture of custom made cranial titanium plates. J. Med. Eng. Technol., 23: 26-28. PMID: 10202700
Yanai, M.D.A., 1991. Resin sealant: A new method of methyl methacrylate cranioplasty. Technical note. J. Neurosurg, 75: 328-330. PMID: 2072176 